\title{
BMJ Open Comparative efficacy and safety of transurethral laser surgery with holmium laser, KTP laser, 2-micron laser or thulium laser for the treatment of non-muscle invasive bladder carcinoma: a protocol of network meta- analysis
}

\author{
Jinfeng Yu, Junbiao Zheng
}

To cite: Yu J, Zheng J. Comparative efficacy and safety of transurethral laser surgery with holmium laser, KTP laser, 2-micron laser or thulium laser for the treatment of non-muscle invasive bladder carcinoma: a protocol of network meta-analysis. BMJ Open 2021;11:e055840. doi:10.1136/ bmjopen-2021-055840

- Prepublication history for this paper is available online. To view these files, please visit the journal online (http://dx.doi. org/10.1136/bmjopen-2021055840).

Received 24 July 2021 Accepted 22 November 2021

Check for updates

(C) Author(s) (or their employer(s)) 2021. Re-use permitted under CC BY-NC. No commercial re-use. See rights and permissions. Published by BMJ.

Department of Urology Surgery, The First People's Hospital of Jiashan, Jiashan, Zhejiang, China

Correspondence to Dr Junbiao Zheng; zhengjb8816@163.com

\section{ABSTRACT}

Introduction The potential of transurethral laser surgery in treating non-muscle invasive bladder cancer (NMIBC) has been confirmed, however which types of lasers may be preferentially prescribed remains a debate. The aim of this network meta-analysis is to investigate the comparative efficacy and safety of transurethral laser surgery with four common types of laser including holmium laser, potassium titanylphosphate (KTP) laser, 2-micron laser or thulium laser for the treatment of NMIBC. Methods and analysis A systematic search will be conducted to search all potentially eligible randomised controlled trials comparing different transurethral laser surgeries with each other or with standard transurethral resection among patients with NMIBC in PubMed, Embase, the Cochrane library, China National Knowledge Infrastructure, Wanfang database and Chongqing VIP from their inception until 31 May 2021. Two reviewers will be asked to independently select eligible studies, and assess the risk of bias of individual study with Cochrane risk of bias assessment tool. A random-effects network metaanalysis based on Markov chain Monte Carlo method will be carried out. Ranking probabilities will be considered to rank all laser types. Quantitative analysis will be carried out by using WinBUGS V.1.4.3.

Ethics and dissemination Ethical approval is not required because this is a network meta-analysis of published data. We will submit all findings to some conferences for preliminary communication and to a peerreviewed journal for publication.

Trial registration number 10.17605/OSF.I0/TD9MW.

\section{INTRODUCTION}

Bladder cancer is one of the most common urological malignant tumour, with an estimated 0.544 million new cases and 0.212 million new death cases worldwide in 2020. ${ }^{1}$ Bladder cancer can be classified into muscle invasive bladder cancer (MIBC)
Strengths and limitations of this study

To the best of the authors' knowledge, this is the first network meta-analysis of investigating comparative efficacy and safety of four common types of lasers in treating non-muscle invasive bladder carcinoma.

- This study may provide the best possible type of laser option and reliable evidence-based medicine for the clinical treatment of non-muscle invasive bladder carcinoma.

- This network meta-analysis also has some limitations, such as publication bias, clinical heterogeneity and selection bias.

and non-muscle invasive bladder cancer (NMIBC), and NMIBC accounts for more than $70 \%$ bladder cancer. ${ }^{2}$ Transurethral resection followed by chemotherapy has been regarded as the standard regime for the treatment of NMIBC; ${ }^{3}{ }^{4}$ however, this technique also faced some technical challenges (eg, difficult control of cutting depth) and also caused several severe complications (eg, obturator nerve reflex, bladder perforation and iliac vascular injury) $)^{56}$ although it has been developed maturely. Meanwhile, it has been reported that transurethral resection was also associated with an increased risk of recurrence owing to the nature of piecemeal resection. ${ }^{7}$ It is therefore essential to explore novel treatment regimes. ${ }^{3}$

It is exciting that, as the laser technology develops, the transurethral laser surgery has been developed and then widely used in clinically treating NMIBC in recent years, with promising effects and safety. ${ }^{8}$ Several original studies have investigated the comparative 


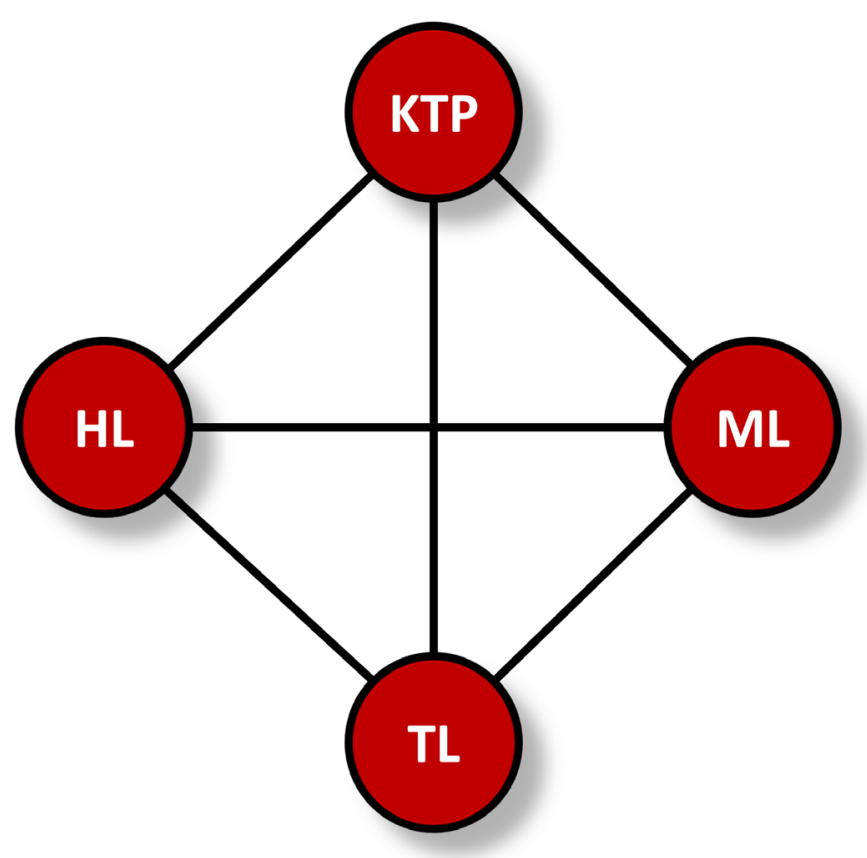

Figure 1 Possible associations of four types of lasers. HL, holmium laser; KTP, potassium titanylphosphate laser; ML, 2-micron laser; TL, thulium laser.

efficacy and safety of transurethral laser surgery and standard transurethral resection, ${ }^{10-13}$ and more importantly, two meta-analyses ${ }^{1415}$ have been performed to establish the clinical value of transurethral laser surgery in treating NMIBC. However, several kind of lasers including holmium laser, potassium titanylphosphate (KTP) laser, 2-micron laser and thulium laser have been found to be useful in transurethral laser therapy for NMIBC, ${ }^{16}$ and the possible associations of these four types of lasers are displayed in figure 1. Although, meta-analysis performed by Xu and colleagues separately investigated the comparative efficacy and safety between different transurethral laser surgeries and standard transurethral resection through performing subgroup analysis ${ }^{15}$ it remains unclear which types of laser should be preferentially selected owing to the nature of conventional direct meta-analysis. ${ }^{17}$

As an expansion of conventional direct meta-analysis, network meta-analysis has the ability of simultaneously comparing multiple treatments at a time. ${ }^{1819}$ Therefore, we designed this systematic review and network metaanalysis to determine the optimal type of laser through investigating the comparative efficacy and safety and calculating rank probabilities of holmium laser, KTP laser, 2-micron laser and thulium laser.

\section{METHODS AND ANALYSIS}

\section{Protocol registration}

We developed this protocol of systematic review and network meta-analysis of randomised controlled trials (RCTs) in accordance with the methodological framework developed by the Cochrane Collaboration. ${ }^{20}$ Moreover, we also followed the recommendations reported in the Preferred Reporting Items for Systematic Review and Meta-Analysis Protocols (PRISMA-P) 2015 statement. ${ }^{21}$ Moreover, we have also released the protocol through registering it on a public platform named as open science framework (OSF) (available at https://osf.io/td9mw). And thus, our study protocol was funded through a protocol registry. No ethical approval and informed consent will be required because all statistical analyses would be conducted on the basis of published studies. The present network meta-analysis will start on 1 October 2021, and will end on 1 May 2022.

\section{Patient and public involvement}

No patients involved.

\section{Eligibility criteria}

Inclusion criteria

We developed the following inclusion criteria: (1) adult patients were definitively confirmed with primary NMIBC; (2) RCTs investigating the comparative efficacy and safety of transurethral laser surgery with different laser types or between transurethral laser surgery and standard transurethral resection; and (3) reported at least one of the following outcomes including operation time, catheterisation time, incidence of obturator nerve reflex, incidence of bladder perforation, length of hospital stay, bladder irritation and recurrence rate.

\section{Exclusion criteria}

We will exclude a study if it covers at least one of the following criteria: (1) the patients were determined to have recurrent or MIBC; (2) sufficient data for statistical analysis is not accessible; and (3) duplicate study with poor quality and insufficient data.

\section{Information sources}

We will assign two independent reviewers to electronically identify potentially eligible studies in PubMed, Embase, the Cochrane Library, China National Knowledge Infrastructure, Wanfang database and Chongqing VIP from their inception until 31 May 2021. We will not impose any restrictions such as publication language and publication status. We will use the following terms to construct the search strategy with the method of combining Medical Subject Headings and full-text word: non-muscle invasive bladder cancer, transurethral resection, laser and random. As an example, we summarised the detailed search strategy of PubMed in table 1. We will also check references of all included studies to include additional studies. We will use the latest PRISMA flow diagram ${ }^{22}$ (V.2020, figure 2) to display the process of identification and selection of study. Any conflicts at this stage will be resolved through consulting a third reviewer.

\section{Study selection and data extraction}

We will first impose all records exported out from target databases into EndNote software, and then duplicate records will be removed through running the function of Finding Duplicate References. Second, we will evaluate 


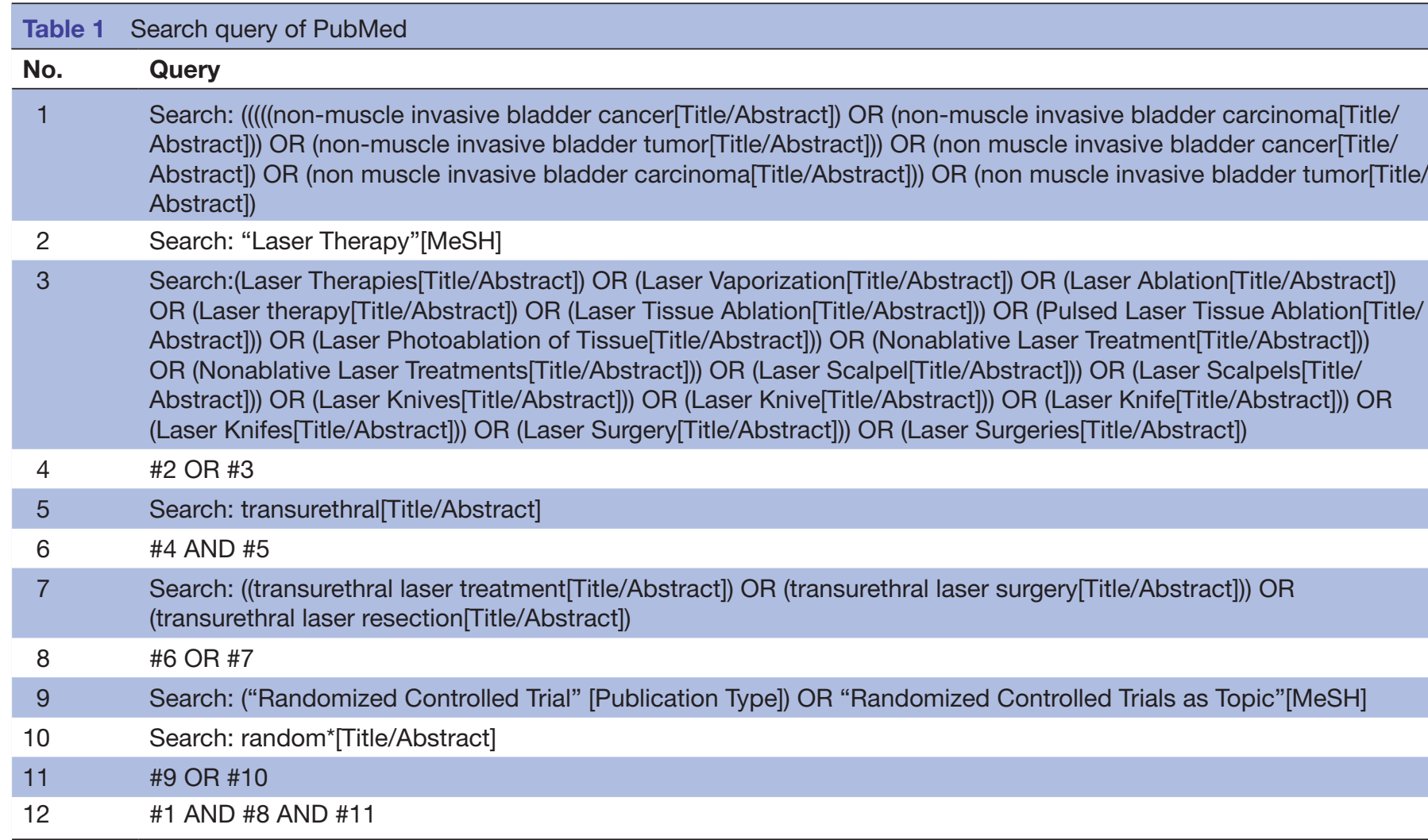

MeSH, Medical Subject Headings.

the eligibility of each record through reviewing title and abstract. Third, we will obtain full-texts of retained records which are selected at the second stage for further evaluating eligibility. W will record the number of excluded studies and corresponding reasons of excluding each study at the third stage.

Two independent reviewers will be assigned to extract the following essential information with the standard data extraction sheet which has been designed by our team: the first author's name, year of publication, country of performing study, age, sample size, gender with proportion of female patients, characteristics of stones, details of risk of bias, outcomes and financial information. We will use the recommended formula to estimate mean and $\mathrm{SD}$ if a continuous variable was reported as median and range or quartile. ${ }^{23}$ We will contact the corresponding author to require information when the essential data are insufficient or not provided in the original study through sending an email. If no information can be added from authors, qualitative method will be used to summarise the findings. Any conflicts at this stage will be resolved through consulting a third reviewer.

\section{Assessment of risk of bias}

We will appoint two reviewers independently assess the methodological quality of each included studies with the Cochrane Risk Bias Assessment Tool. ${ }^{24}$ With this tool, the methodological quality of the individual study will be assessed from seven items as follows: random sequence generation, allocation concealment, blinding of participants and personnel, blinding of outcome assessor, incomplete data, selective reporting and other bias sources. Each item will be classified as low, unclear or high risk of bias according to the assessment criteria. The overall methodological quality of individual study will be rated as low if at least one item was labelled with high risk of bias, high if all items were labelled with low risk of bias or moderate if at least one item was labelled with unclear risk of bias but no item was labelled with high risk of bias. We will use Microsoft Word software to make risk of bias summary (table 2). Any conflicts about risk of bias assessment will be settled by consulting a third reviewer.

\section{Statistical analysis}

We will first conduct a conventional pairwise meta-analysis based on a random-effects model for all outcomes. We will use the OR with 95\% CI to express dichotomous variables, and use mean difference (MD) or standard MD (SMD) with $95 \% \mathrm{CI}$ to express continuous variables. After performing statistical analysis, we will first evaluate the heterogeneity across studies. Cochrane $Q$ statistic (based on $\chi^{2}$ test ${ }^{25}$ and $\mathrm{I}^{2}$ statistic $^{26}$ will be used to qualitatively and quantitatively assess heterogeneity, respectively. Being dependent on the criteria, a $\mathrm{I}^{2}>50 \%$ and $\mathrm{p}$ value $<0.1$ suggests a substantial heterogeneity. However, we will select statistical model according to the level of heterogeneity because variations across studies cannot prevent that in the real world. Direct meta-analysis will be conducted by using RevMan V.5.3 software. 


\section{Identification of studies via databases and registers}

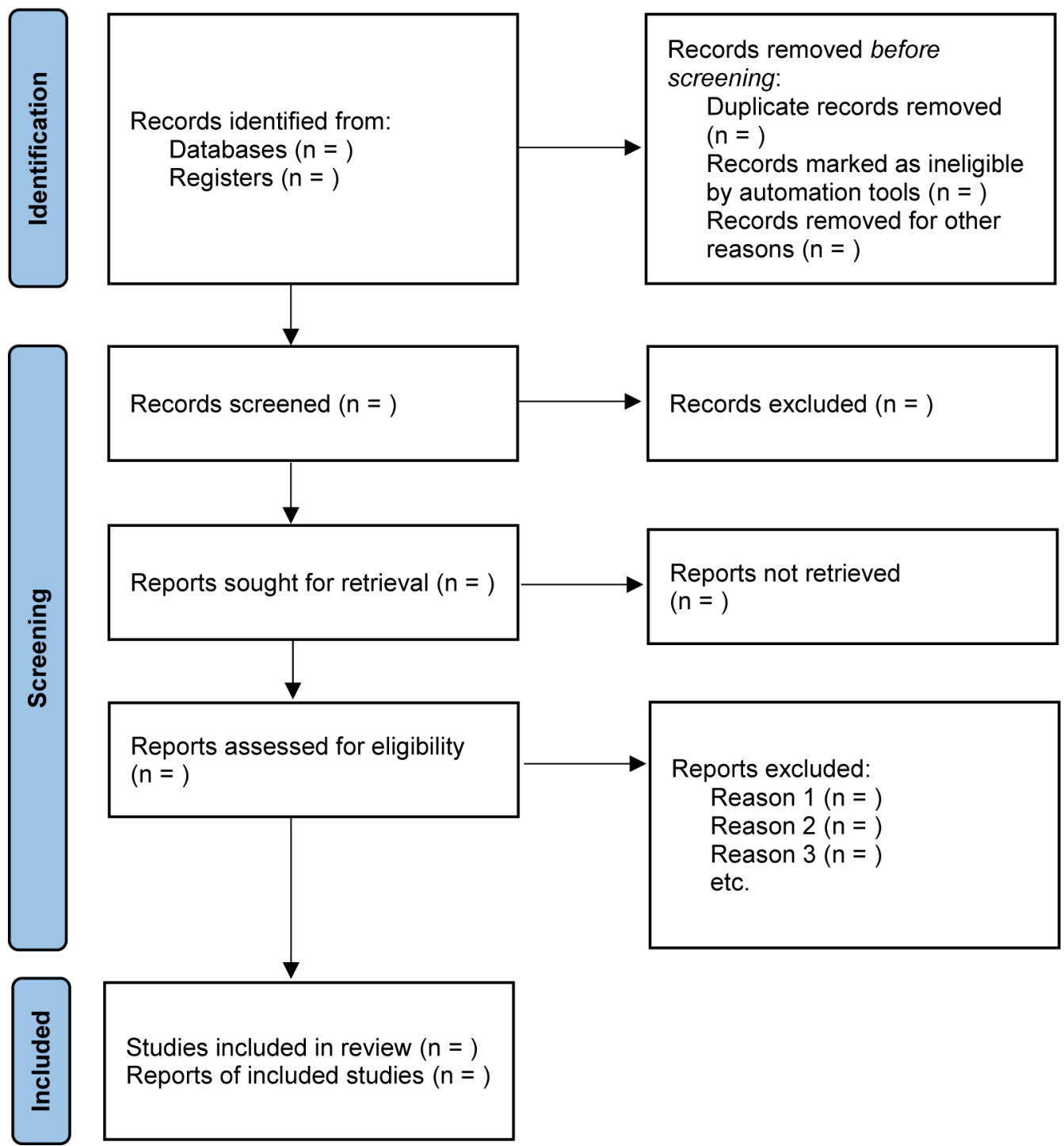

Figure 2 Preferred Reporting Items for Systematic Review and Meta-Analysis flow diagram of study identification and selection.

After completing conventional pairwise meta-analysis, we will subsequently conduct a random-effects network meta-analysis by using Bayesian Markov-chain Monte
Carlo method, which will be done with WinBUGS V.1.4.3. ${ }^{18}$ All estimates in network meta-analysis will be expressed as OR or SMD with $95 \%$ creditable interval.

Table 2 Risk of bias summary

\begin{tabular}{|c|c|c|c|c|c|c|}
\hline $\begin{array}{l}\text { Random } \\
\text { sequence } \\
\text { generation } \\
\text { (selection } \\
\text { bias) }\end{array}$ & $\begin{array}{l}\text { Allocation } \\
\text { concealment } \\
\text { (selection bias) }\end{array}$ & $\begin{array}{l}\text { Blinding of } \\
\text { personnels and } \\
\text { participants } \\
\text { (performance } \\
\text { bias) }\end{array}$ & $\begin{array}{l}\text { Blinding of } \\
\text { outcome } \\
\text { assessor } \\
\text { (detection } \\
\text { bias) }\end{array}$ & $\begin{array}{l}\text { Incomplete } \\
\text { data (attrition } \\
\text { bias) }\end{array}$ & $\begin{array}{l}\text { Selective } \\
\text { reporting } \\
\text { (reporting } \\
\text { bias) }\end{array}$ & $\begin{array}{l}\text { Other } \\
\text { bias }\end{array}$ \\
\hline
\end{tabular}

Study 1

Study 2

Study 3

Study 4

Study 5 
The convergence of data will be evaluated by using the Brooks Gelman-Rubin statistical method, and a potential proportional reduction factor of closing to 1 indicating more reliable convergence. ${ }^{27} 28$ We will calculate the surface under the cumulative ranking curve to determine relatively optimal frequency, ${ }^{29}$ and a value of closing to 1 for a certain frequency regime indicates that it is more likely to be the best option. Moreover, we will also use node-split method to test whether the presence or not of inconsistency between direct and indirect evidence when a closed loop was available in network meta-analysis,${ }^{30}$ and a $\mathrm{p}$ value $<0.05$ indicates the presence of inconsistency. ${ }^{17}$ All estimates in network meta-analysis will be graphically shown by using Excel. We will also conduct sensitivity analysis to examine the robustness of pooled results by using the method of one-study remove. ${ }^{31}$

\section{Subgroup and sensitivity analyses}

If there is heterogeneity and the data are sufficient, we will conduct subgroup analysis to determine the potential reasons causing heterogeneity and investigate the comparative efficacy of each group. Data may be compared between patients of different course of disease and treatment duration. Moreover, we will also conduct sensitivity analysis through removing studies with high risk of bias when there is insufficient number of eligible studies for individual comparison. We will determine the robustness of pooled results through comparing the consistency between original results and pooled result of sensitivity analysis.

\section{Publication bias}

We will draw comparison-adjusted funnel plot to qualitative inspect whether presence or not of publication bias if the accumulated number of eligible studies was more than 10 for individual comparison, ${ }^{32}$ and an asymmetric funnel plot indicates the presence of publication bias. ${ }^{33}$

\section{DISCUSSION}

Although standard transurethral resection has been regarded as the standard regime for the treatment of NMIBC, ${ }^{34}$ several technique challenges and severe complications limited its application. ${ }^{5}$ Numerous efforts have been made for the purpose of developing novel technologies. It is exciting that development of laser technology accelerate the application of laser surgery in clinical practice. ${ }^{8}$ Several original studies have been performed to investigate the comparative efficacy and safety between transurethral laser surgery and standard transurethral resection in treating NMIBC, and two recent metaanalyses further established the advantages of transurethral laser surgery for the treatment of NMIBC related to standard transurethral resection. ${ }^{1415}$ Four types of lasers have been widely used in transurethral laser therapy for NMIBC, ${ }^{16}$ however it is unclear which types of laser may be optimal because previous direct meta-analysis only separately compared individual type of laser with standard transurethral resection by conducting subgroup analysis. ${ }^{15}$ Certainly, it cannot obtain comprehensive comparative efficacy and safety of four types of laser due to the nature of conventional direct meta-analysis. ${ }^{17}$ Therefore, it is imperative to conduct a network meta-analysis which is an expansion of conventional direct meta-analysis and has the ability of simultaneously comparing multiple treatments (more than two) at one time and calculating rank probabilities of all treatments ${ }^{19}$ to further determine the optimal type of laser for the treatment of NMIBC.

The present systematic review and network metaanalysis will first investigate the comparative efficacy and safety of transurethral laser surgery with different laser types for the treatment of NMIBC. Two methodological strengths should be emphasised in this systematic review and network meta-analysis: (1) we will conduct a comprehensive literature based on highly sensitive search strategy and (2) ranking probabilities of four laser types will be estimated and then determine the optimal transurethral laser surgery.

We developed this systematic review and network metaanalysis to first investigate the comparative efficacy and safety of transurethral laser surgery with different laser types for the treatment of NMIBC. After completing this systematic review and network meta-analysis, we will obtain more reliable and robust findings which will provide more accurate and reliable evidence for making decisions for the treatment of NMIBC in clinical practice.

Acknowledgements We would like to deeply appreciate the open science framework (OSF) for acceptance of our application of registering this protocol.

Contributors JY and JZ developed and designed this protocol. JY and JZ developed search query and contributed to the methodological development of the protocol. JY drafted the manuscript and other authors critically made a revision. All authors reviewed and approved the final version for publication. JZ is the review guarantor.

Funding The current review is funded by the open science framework (OSF) registry.

Disclaimer Funders and sponsors have no role in the design of this protocol. Competing interests None declared.

Patient and public involvement Patients and/or the public were not involved in the design, or conduct, or reporting, or dissemination plans of this research.

Patient consent for publication Not applicable.

Provenance and peer review Not commissioned; externally peer reviewed.

Open access This is an open access article distributed in accordance with the Creative Commons Attribution Non Commercial (CC BY-NC 4.0) license, which permits others to distribute, remix, adapt, build upon this work non-commercially, and license their derivative works on different terms, provided the original work is properly cited, appropriate credit is given, any changes made indicated, and the use is non-commercial. See: http://creativecommons.org/licenses/by-nc/4.0/.

ORCID iD

Junbiao Zheng http://orcid.org/0000-0002-0228-4946

\section{REFERENCES}

1 Sung H, Ferlay J, Siegel RL, et al. Global cancer statistics 2020: GLOBOCAN estimates of incidence and mortality worldwide for 36 cancers in 185 countries. CA Cancer J Clin 2021;71:209-49.

2 Kamat AM, Hahn NM, Efstathiou JA, et al. Bladder cancer. Lancet 2016;388:2796-810. 
3 Babjuk M, Böhle A, Burger M, et al. EAU guidelines on non-muscleinvasive urothelial carcinoma of the bladder: update 2016. Eur Urol 2017;71:447-61.

4 Nishiyama $\mathrm{H}$. Asia consensus statement on NCCN clinical practice guideline for bladder cancer. Jpn J Clin Oncol 2018;48:3-6.

5 Gregg JR, McCormick B, Wang L, et al. Short term complications from transurethral resection of bladder tumor. Can J Urol 2016;23:8198-203.

6 Shah NF, Sofi KP, Nengroo SH. Obturator nerve block in transurethral resection of bladder tumor: a comparison of ultrasound-guided technique versus ultrasound with nerve stimulation technique. Anesth Essays Res 2017;11:411-5.

7 Koie T, Ohyama C, Hosogoe S, et al. Oncological outcomes of a single but extensive transurethral resection followed by appropriate intra-vesical instillation therapy for newly diagnosed non-muscleinvasive bladder cancer. Int Urol Nephrol 2015;47:1509-14.

8 Korn SM, Hübner NA, Seitz C, et al. Role of lasers in urology. Photochem Photobiol Sci 2019;18:295-303.

9 Zhang J, Wang L, Mao S, et al. Transurethral en bloc resection with bipolar button electrode for non-muscle invasive bladder cancer. Int Urol Nephrol 2018;50:619-23.

10 Chen J, Zhao Y, Wang S, et al. Green-light laser en bloc resection for primary non-muscle-invasive bladder tumor versus transurethral electroresection: a prospective, nonrandomized two-center trial with 36-month follow-up. Lasers Surg Med 2016;48:859-65.

11 Zhang X-R, Feng C, Zhu W-D, et al. Two micrometer continuouswave Thulium laser treating primary non-muscle-invasive bladder cancer: is it feasible? A randomized prospective study. Photomed Laser Surg 2015;33:517-23.

12 Xu Y, Guan W, Chen W, et al. Comparing the treatment outcomes of potassium-titanyl-phosphate laser vaporization and transurethra electroresection for primary nonmuscle-invasive bladder cancer: a prospective, randomized study. Lasers Surg Med 2015;47:306-11.

13 Chen X, Liao J, Chen L, et al. En bloc transurethral resection with 2-micron continuous-wave laser for primary non-muscle-invasive bladder cancer: a randomized controlled trial. World J Urol 2015;33:989-95.

$14 \mathrm{Li} \mathrm{C}$, Gao L, Zhang J, et al. The effect of holmium laser resection versus standard transurethral resection on non-muscle-invasive bladder cancer: a systematic review and meta-analysis. Lasers Med Sci 2020;35:1025-34.

$15 \mathrm{Xu}$ J, Wang C, Ouyang J, et al. Efficacy and safety of transurethral laser surgery versus transurethral resection for non-muscle-invasive bladder cancer: a meta-analysis and systematic review. Urol Int 2020;104:810-23.

16 Enikeev D, Shariat SF, Taratkin M, et al. The changing role of lasers in urologic surgery. Curr Opin Urol 2020;30:24-9.

17 Albert I, Makowski D. Ranking crop species using mixed treatment comparisons. Res Synth Methods 2019;10:343-59.
18 Lu G, Ades AE. Combination of direct and indirect evidence in mixed treatment comparisons. Stat Med 2004;23:3105-24.

19 Cipriani A, Higgins JPT, Geddes JR, et al. Conceptual and technical challenges in network meta-analysis. Ann Intern Med 2013;159:130-7.

20 Higgins JPT, Altman DG, Sterne JAC. Cochrane Handbook for systematic reviews of interventions. Cochrane, 2011.

21 Moher D, Shamseer L, Clarke M, et al. Preferred reporting items for systematic review and meta-analysis protocols (PRISMA-P) 2015 statement. Syst Rev 2015;4:1.

22 Page MJ, McKenzie JE, Bossuyt PM, et al. The PRISMA 2020 statement: an updated guideline for reporting systematic reviews. BMJ 2021;372:n71.

23 Wan X, Wang W, Liu J, et al. Estimating the sample mean and standard deviation from the sample size, median, range and/or interquartile range. BMC Med Res Methodol 2014;14:135.

24 Higgins JPT, Altman DG, Gøtzsche PC, et al. The Cochrane collaboration's tool for assessing risk of bias in randomised trials. BMJ 2011;343:d5928.

25 Bowden J, Tierney JF, Copas AJ, et al. Quantifying, displaying and accounting for heterogeneity in the meta-analysis of RCTs using standard and generalised Q statistics. BMC Med Res Methodol 2011;11:41

26 Higgins JPT, Thompson SG. Quantifying heterogeneity in a metaanalysis. Stat Med 2002;21:1539-58.

27 Brooks S, Gelman A. General methods for monitoring convergence of iterative simulations. J Comput Graphi Stat 1998;7:434-55.

28 Burger DA, Schall R. A Bayesian nonlinear mixed-effects regression model for the characterization of early bactericidal activity of tuberculosis drugs. J Biopharm Stat 2015;25:1247-71.

29 Salanti G, Ades AE, loannidis JPA. Graphical methods and numerical summaries for presenting results from multipletreatment meta-analysis: an overview and tutorial. $J$ Clin Epidemiol 2011;64:163-71.

30 Dias S, Welton NJ, Caldwell DM, et al. Checking consistency in mixed treatment comparison meta-analysis. Stat Med 2010;29:932-44.

31 Carpenter J, Rücker G, Schwarzer G. Assessing the sensitivity of meta-analysis to selection bias: a multiple imputation approach. Biometrics 2011;67:1066-72.

32 Palma Pérez S, Delgado Rodríguez M. [Practical considerations on detection of publication bias]. Gac Sanit 2006;20(Suppl 3):10-16.

33 Page MJ, McKenzie JE, Higgins JPT. Tools for assessing risk of reporting biases in studies and syntheses of studies: a systematic review. BMJ Open 2018;8:e019703.

34 Sexton WJ, Wiegand LR, Correa JJ, et al. Bladder cancer: a review of non-muscle invasive disease. Cancer Control 2010;17:256-68. 